\title{
Catalysis of the Electroreduction of Allyl Chloride by Cobalt 2,2'-Bipyridine Complexes
}

\author{
Shlomo Margel and Fred C. Anson* \\ Arthur Amos Noyes Laboratory, California Institute of Technology, Pasadena, California 91125
}

\begin{abstract}
The electrochemical reduction of allyl chloride is strongly catalyzed in the presence of cobalt complexes of 2,2'-bipyridine. A prominent reaction product of the catalyzed reduction is 1,5-hexadiene. Voltammetry, coulometry, and gas chromatographic data are presented and analyzed and a mechanistic scheme proposed to account for the catalytic action of the cobalt-2,2'-bipyridine complexes.
\end{abstract}

Recently we reported studies (1) which examined a previous claim (2) that the electroreduction of acrylonitrile in acetonitrile was catalyzed by a complex of cobalt with $2,2^{\prime}$-bipyridine. These studies demonstrated the absence of such catalysis for acrylonitrile and a number of other vinyl monomers but one substrate, allyl chloride, did exhibit a very large enhancement in its reduction rate in the presence of cobalt $2,2^{\prime}-\mathrm{bi}-$ pyridine complexes. This report summarizes our experimental observations with this system and presents a possible reaction scheme to account for the observed catalysis.

\section{Experimental}

Reagents.-The solvent was prepared from spectroscopic grade acetonitrile which was stirred over $\mathrm{CaH}_{2}$ for $24 \mathrm{hr}$, distilled near room temperature under reduced pressure, and stored under argon. Polarographic grade tetraethylammonium perchlorate (TEAP) (Southwestern Analytical Company) was vacuum dried and used as the supporting electrolyte without additional purification.

$\mathrm{Co}(\text { bipy })_{3}\left(\mathrm{ClO}_{4}\right)_{3}, \mathrm{Co}(\text { bipy })_{3}\left(\mathrm{ClO}_{4}\right)_{2}$ (bipy $\equiv 2,2$ 'bipyridine), and $\mathrm{Co}(\text { phen })_{3}\left(\mathrm{ClO}_{4}\right)_{2}$ (phen $\equiv 1,10-$ phenanthroline) were prepared as previously described (1). Solutions of $\mathrm{Co}$ (bipy $)_{2}\left(\mathrm{ClO}_{4}\right)_{2}$ were prepared by mixing $\mathrm{Co}\left(\mathrm{ClO}_{4}\right)_{2}$ and $2,2^{\prime}$-bipyridine in a molar ratio of 1:2 in acetonitrile. Allyl chloride (Eastman) and allyl alcohol (Aldrich) were distilled just prior to their use.

Apparatus.-Cyclic voltammograms were obtained with a Princeton Applied Research (PAR) Model 173 potentiostat driven by a PAR Model 175 programmer. Current-voltage curves were recorded with a Tektronix Model 564 storage oscilloscope and photographed with a Tetronix Model C-12 camera. Controlled potential coulometry was conducted with the same potentiostat equipped with a Model 179 digital coulometer. A three-compartment electrochemical cell was employed with double isolation of the $\mathrm{Ag} / 0.1 \mathrm{M} \mathrm{Ag}^{+}$ reference electrode against which all potentials are quoted. Solutions were deoxygenated with prepurified argon which was passed successively through aqueous chromous chloride solution, a calcium chloride drying tower, and purified acetonitrile before entering the test solution. Concentrations of allyl chloride and 1,5hexadiene were monitored gas chromatographically with a Hewlett-Packard Model 5830 chromatograph using a $12 \mathrm{ft}$ column packed with Carbowax 20M on Chromosorb $\mathrm{W}$.

\section{Results}

Of the three characteristic voltammetric waves exhibited by Co (bipy) $3^{3+}$ in acetonitrile $(1,2)$ the second is the first to be affected by the presence of allyl chloride. In addition, as was true for acrylonitrile (1), a new wave appears between the original second and

* Electrochemical Society Active Member.

Key words: catalysis, $\pi$-coordination, intramolecular. third waves. These features are shown in Fig. 1. The peak current of the new wave with a peak potential near $-1500 \mathrm{mV}$ increases at the expense of the third wave $\left(E_{\mathrm{p}}=-1950 \mathrm{mV}\right)$ as the concentration of added allyl chloride is increased (Fig. 1). At a fixed concentration of allyl chloride the ratio of peak current to square root of scan rate for the new wave at first increases as the scan rate decreases (Fig. 2) but eventually decreases again at very low scan rates. The magnitude of the wave is also suppressed by the presence of excess 2,2'-bipyridine (Fig. 2).

The behavior is rather similar to that obtained with acrylonitrile and is consistent with an analogous interpretation of the origin of the new wave (1): It corresponds to the reduction of a mixed allyl chloride, $2,2^{\prime}$-bipyridine complex of cobalt(I) that is the product of reaction [1]

$$
\begin{aligned}
\mathrm{Co}(\text { bipy })_{3}+ & +\mathrm{CH}_{2} \mathrm{CHCH}_{2} \mathrm{Cl} \\
& \rightleftharpoons \mathrm{Co}(\text { bipy })_{2}\left(\mathrm{CH}_{2} \mathrm{CHCH}_{2} \mathrm{Cl}\right)++ \text { bipy }
\end{aligned}
$$

However, in contrast with the cobalt(I) acrylonitrile system, the mixed complex with allyl chloride is unstable with respect to intramolecular reduction of the coordinated allyl chloride by the cobalt(I). This reaction, which results in the regeneration of cobalt (II) at the electrode surface, is the reason that the addition of allyl chloride causes the peak current of the wave corresponding to the reduction of cobalt(II) to cobalt(I) to exceed its value in the absence of allyl chloride (Fig. 2) except at scan rates high enough to prevent reaction [1] (and the subsequent intramolec-

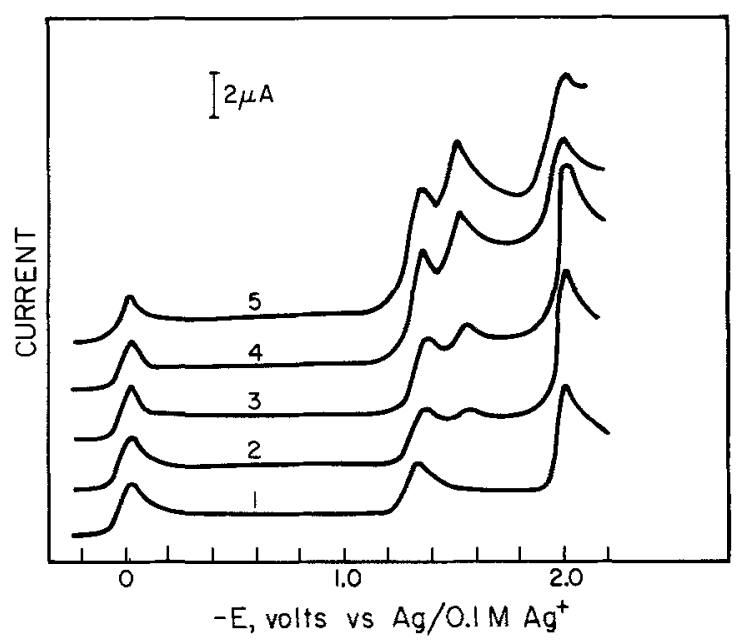

Fig. 1. Effect of allyl chloride on linear scan voltammograms for $1 \mathrm{mM} \mathrm{Co}(\text { bipy })_{3}{ }^{3+}$ in acetonitrile. Allyl chloride concentrations: curve 1, 0 ; curve 2, $1 \mathrm{mM}$; curve $3,5 \mathrm{mM}$; curve 4, 30 $\mathrm{mM}$; curve $5,0.1 \mathrm{M}$. Supporting electrolyte: $0.1 \mathrm{M}$. TEAP. Scan rate: $0.5 \vee \mathrm{sec}^{-1}$. Platinum electrode area: $0.43 \mathrm{~cm}^{2}$. 


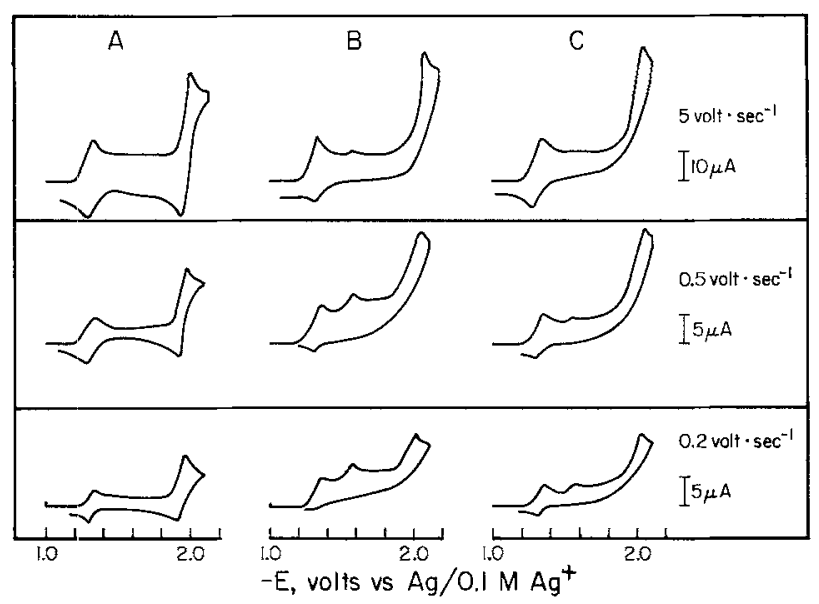

Fig. 2. Effect of allyl chloride on cyclic voltommograms for $\mathbf{0 . 5}$ $\mathrm{mM} \mathrm{Co}(\text { bipy })_{3}{ }^{2+}$ in acetonitrile. Allyl chloride concentrations:

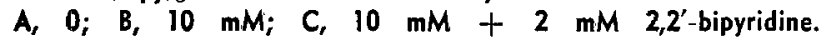
Supporting electrolyte: 0.1M TEAP. Platinum electrode area: 1.6 $\mathrm{cm}^{2}$.

ular reduction reaction) from proceeding significantly from left to right.

The instability of the mixed cobalt(I)-allyl chloride complex made it impossible to evaluate the equilibrium constant for reaction [1] from cyclic voltammetric data as was done for the acrylonitrile complex. However, from the qualitative observation that larger concentrations of allyl chloride than of acrylonitrile are required to cause the formation of the new wave, it seems likely that the equilibrium constant is smaller for the allyl chloride complex.

The voltammograms in Fig. 3 show that substitution of $\mathrm{Co}$ (bipy) ${ }_{2}{ }^{+}$for Co (bipy) ${ }_{3}{ }^{+}$significantly increases the rate at which reaction [1] proceeds from left to right (the same is true for the analogous reaction involving acrylonitrile). In the case of $\mathrm{Co}$ (bipy) ${ }_{3}{ }^{+}$ the slow step is apparently the loss of one of the coordinated 2,2'-bipyridine ligands.

No evidence of the formation of a mixed complex was obtained when $\mathrm{Co}(\text { bipy })_{3}{ }^{+}$was exposed to ally] alcohol or when $\mathrm{Co}$ (phen) ${ }_{3}{ }^{+}$was exposed to even large excesses of allyl chloride.

Note that the rate of the reduction of the coordinated ally1 chloride is apparently slower than the rate at which it coordinates to $\mathrm{Co}$ (bipy) ${ }_{3}{ }^{+}$or $\mathrm{Co}(\text { bipy })_{2}+$. Otherwise, the new wave in Fig. 2 and 3 arising from the reduction of the mixed complex would not appear. Instead, the wave corresponding to the reduction of cobalt (II) to cobalt (I) would merely increase in magnitude as all of the allyl chloride was reduced.

The ratio of peak current to square root of scan rate for the reduction of $\mathrm{Co}(\text { bipy })_{3}^{2+}$ to $\mathrm{Co}(\text { bipy })_{3}+$ be-

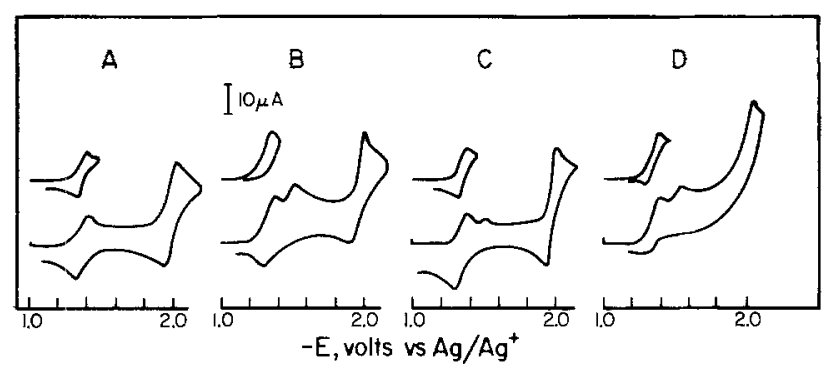

Fig. 3. Comparison of cyclic voltammograms for $\mathrm{Co}(\text { bipy })_{3}{ }^{2+}$ and $\mathrm{Co}_{0}$ (bipy) $)_{2}{ }^{+}$in the presence of allyl chloride. Concentrations of cobalt complex: $1.0 \mathrm{mM} ; \mathrm{A}, \mathrm{Co}$ (bipy) $2_{2}{ }^{2+} ; \mathrm{B}, \mathrm{Co}(\text { bipy })_{2}{ }^{2+}+$ $1 \mathrm{mM}$ allyl chloride; C, $\left.\mathrm{Co}_{\text {(bipy }}\right)^{2+}+1 \mathrm{mM}$ allyl chloride; D, Co(bipy) $3^{2+}+11 \mathrm{mM}$ allyl chloride. The small upper voltammograms in each case show the response obtained if the potential scan is reversed just after the first (Co(II) $\rightarrow \mathrm{Co}_{(1))}$ peak. Scan rate: $0.5 \mathrm{~V} \mathrm{sec}^{-1}$. Other conditions as in Fig. 2.

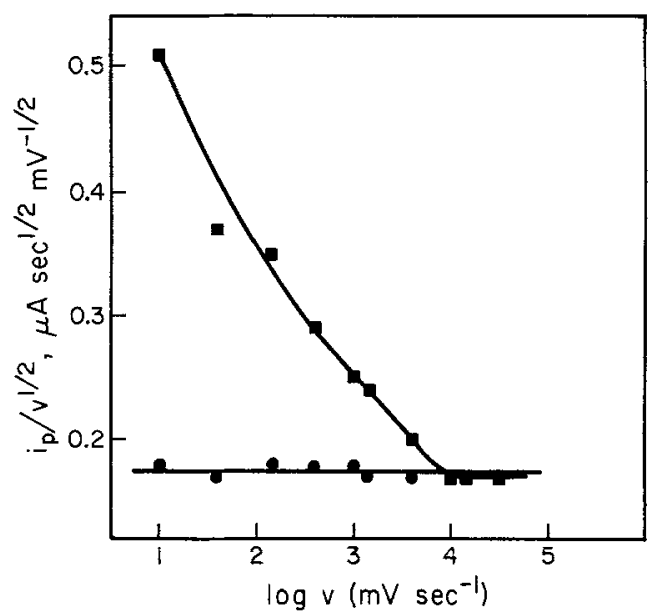

Fig. 4. Scan rate dependence of the ratio of voltammetric peak current, $i_{p}$, to square-root of scan rate for reduction of $\mathrm{Co}_{0}(\text { bipy })_{3}{ }^{2+}$ to $C_{0}(\text { bipy })_{3}{ }^{+} .0,0.5 \mathrm{mM} \mathrm{Co}(\text { bipy })_{3}{ }^{2+} ; \quad, 0.5 \mathrm{mM}$

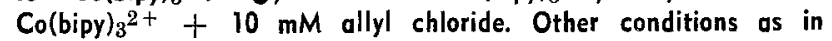
Fig. 2.

comes strongly dependent on scan rate in the presence of allyl chloride as shown in Fig. 4. Only at scan rates high enough to prevent reaction [1] from proceeding does the ratio of $i_{\mathrm{p}}$ to $v^{1 / 2}$ decrease to its value in the absence of allyl chloride.

The behavior of the anodic half of the cyclic voltammograms in Fig. 2 and 3 is also understandable on this basis: Little or no wave is observed for the oxidation of $\mathrm{Co}$ (bipy) ${ }_{3}{ }^{+}$and $\mathrm{Co}$ (bipy) ${ }_{2}{ }^{+}$under conditions where the mixed complex is formed because the product of its further reduction is apparently not oxidizable. The anodic wave for $\mathrm{Co}(\mathrm{I}) \rightarrow \mathrm{Co}$ (II) appears only under conditions where most of any allyl chloride present has been consumed so that $\mathrm{Co}(\text { bipy })_{3}{ }^{+}$or $\mathrm{Co}(\text { bipy })_{2}{ }^{+}$ can persist at the electrode surface and be reoxidized.

By using a mercury electrode it proved possible to use the anodic depolarization of mercury at $+400 \mathrm{mV}$ to monitor the concentration of chloride ion released as the allyl chloride was reduced. There was a good correlation between the magnitude of the anodic wave due to chloride and the increase in the peak current of the wave for the reduction of cobalt (II) to cobalt (I).

\section{Controlled Potential Coulometry}

Electrolysis of solutions of Co(bipy) $3^{2+}$ in acetonitrile at $-1400 \mathrm{mV}$ results in the quantitative formation of the intensely blue Co(bipy) ${ }_{3}+$ ion (1). If allyl chloride is present during such an electrolysis, the deep blue color does not develop during the initial stages of the electrolysis. Gas chromatographic analysis of the solution shows that the allyl chloride is consumed and 1,5-hexadiene is produced as the electrolysis proceeds (Fig. 5). The blue color of the Co (bipy) ${ }_{3}^{+}$ ion persists in the solution only after all of the allyl chloride has been removed. The yield of 1,5 -hexadiene and the quantity of charge required to reduce the allyl chloride are functions of the relative amounts of allyl chloride and cobalt complex present. Table I summarizes the coulometric data. When the ratio of allyl chloride to $\mathrm{Co}$ (bipy) ${ }_{3}{ }^{+}$is small, e.g., experiments 1 and 2 in Table $I$, the reduction consumes precisely one electron per molecule of allyl chloride and a prominent reaction product is 1,5 -hexadiene (the other reaction products were not determined). As this ratio increases allyl chloride disappears from the solution before one electron per molecule is consumed (experiments 3 to 7, Table I), the more so the higher the concentration of allyl chloride. Gas chromatograms of the solutions resulting from such electrolyses (Fig. 6) contain a series of ill-defined peaks corresponding to components with high retention times as would be expected for oligomers of allyl chloride. 


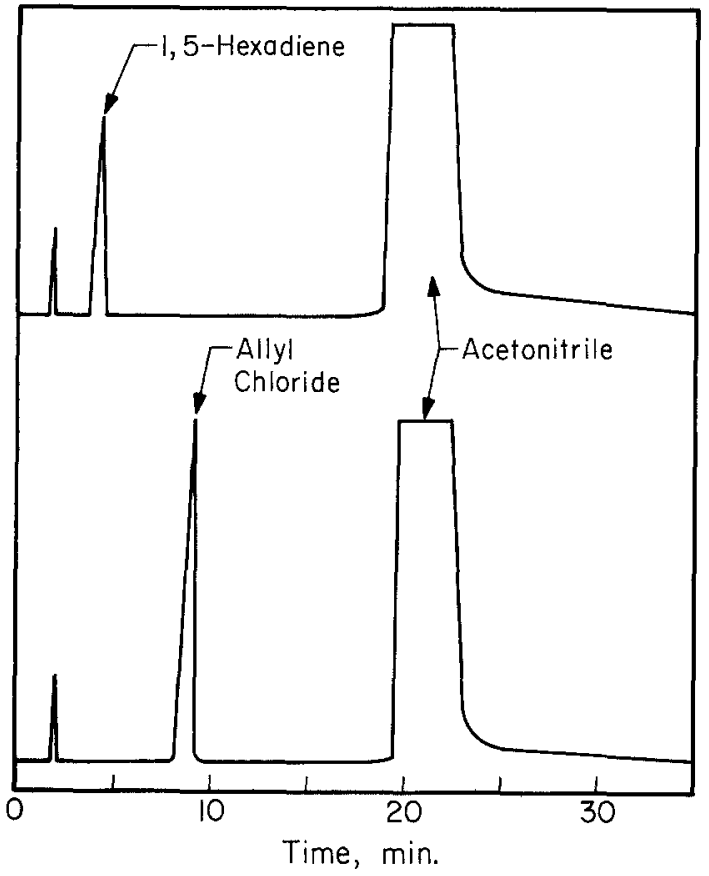

Fig. 5. Gas chromatograms of electrolysis solution before (lower chromatogram) and after (upper chromatogram) exhaustive electrolysis at $-1.4 \mathrm{~V}$ vs. $\mathrm{Ag} / 0.1 \mathrm{M} \mathrm{Ag} \mathrm{At}^{+}$at a platinum gauze electrode. Initial solution composition: $10 \mathrm{mM} \mathrm{Co(bipy)})_{3}^{2+}, 10$ $\mathrm{mM}$ allyl chloride, 0.2M TEAP. The small peak with a retention time near 3 min was obtained in all chromatograms and may be an impurity in the solvent.

During the electrolysis for experiment 7 in Table I a small amount of a white, pastelike solid precipitated from the solution. The solid was collected, washed with water, and vacuum dried at room temperature. Elemental analyses of the solid showed: (percentages) $\mathrm{C}$; $47.5 ; \mathrm{H}, 6.3 ; \mathrm{Cl}, 44$. Calculated for $\left(\mathrm{CH}_{2} \mathrm{CHCH}_{2} \mathrm{Cl}\right)_{n}$ : $\mathrm{C}$, $47.1 ; \mathrm{H}, 6.5 ; \mathrm{Cl}$, 46.4. Thus, at high ratios of allyl chloride to cobalt(I) polymerization of the allyl chloride ensues and is the major pathway by which it is removed from electrolysis solutions.

It is not necessary for the allyl chloride to be present during the electrolytic reduction of $\mathrm{Co}$ (bipy) $3^{2+}$ to $\mathrm{Co}$ (bipy) ${ }_{3}{ }^{+}$in order to achieve the reduction of allyl chloride. For example, the addition, under argon, of $0.1 \mathrm{mM}$ of allyl chloride to $10 \mathrm{ml}$ of a $10 \mathrm{mM}$ solution of $\mathrm{Co}$ (bipy) ${ }_{3}{ }^{+}$prepared by the electrolytic reduction of $\mathrm{Co}$ (bipy) ${ }_{3}{ }^{2}+$ resulted in the rapid fading

Table I. Coulometric data for the catalyzed reduction of a!lyl chloride in the presence of $\mathrm{Co}_{0}(\mathrm{bipy})_{3}{ }^{+*}$

\begin{tabular}{ccccc}
\hline Expt. No. & $\begin{array}{c}{\left[\mathrm{Co}(\text { bipy }) 3^{2+}\right]} \\
(\mathrm{min})\end{array}$ & $\begin{array}{c}\left.\mathrm{CH}_{2} \mathrm{CHCH}_{2} \mathrm{Cl}\right] \\
(\mathrm{mM})\end{array}$ & $\begin{array}{c}\text { Yield of } \\
n_{\text {app }} * *\end{array}$ & $\begin{array}{c}\text { 1,5 hexa- } \\
\text { diene }(\%)\end{array}$ \\
\hline 1 & 10 & 5 & 1.0 & 53 \\
2 & 10 & 10 & 1.0 & 50 \\
3 & 2 & 10 & 0.89 & 41 \\
4 & 2 & 30 & 0.63 & 26 \\
5 & 2 & 100 & 0.32 & 4 \\
6 & 2 & 500 & 0.11 & $\sim 1$ \\
7 & 4 & 5700 & 0.01 & $\sim 1$ \\
8 & 0 & $10 \pm$ & 2.0 & \\
\hline
\end{tabular}

* Reduction at a platinum gauze electrode maintained at -1400 $\mathrm{mV}$ vs. $\mathrm{Ag} / 0.1 \mathrm{M} \mathrm{Ag} \mathrm{Ag}^{+}$in acetonitrile. Supporting electrolyte: $0.1 \mathrm{M}$ tetraethylammonium perchlorate; solution volume: $10 \mathrm{ml}$.

* napp is the ratio of the charge consumed up to the point at which the blue color of Co(bipy) $3^{+}$first appeared to the calculated charge for the one-electron reduction of the allyl chloride present.

The fate of the reduced allyl chloride which did not appear as 1,5-hexadiene was not determined.

Electrode potential was $-2600 \mathrm{mV}$. be reduced to a mixture of 1,5-hexadiene and 1-propene under these conditions $(3,4)$.

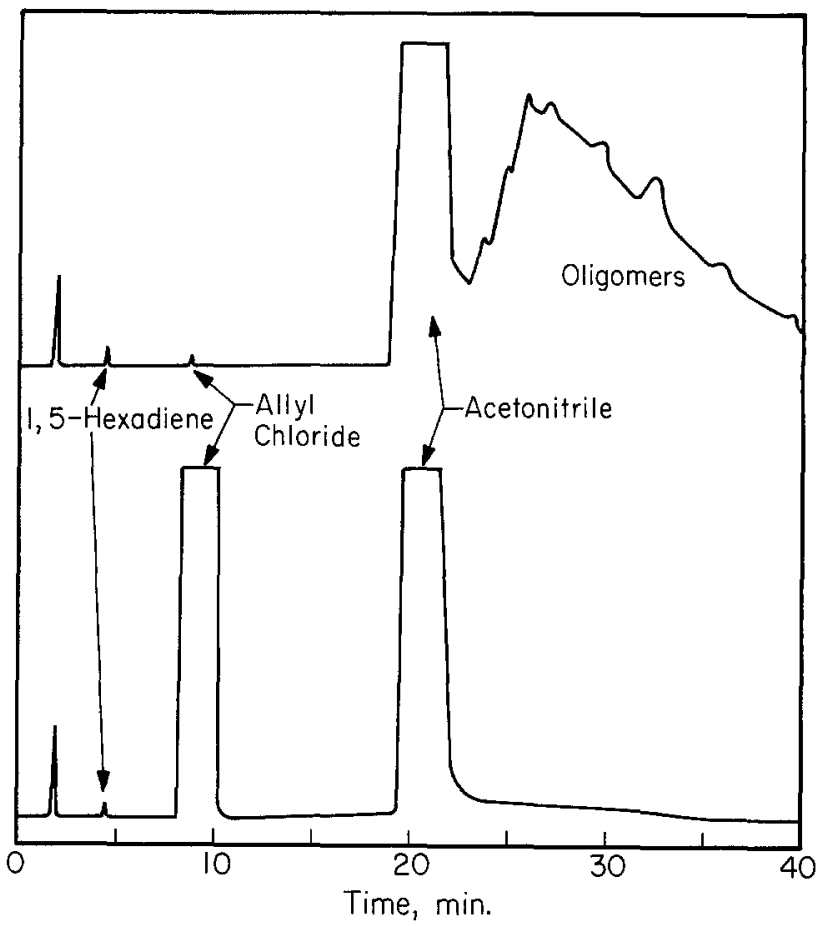

Fig. 6. Gas chromatograms of electrolysis solutions before (lower chromatogram) and after (upper chromatogram) exhaustive electrolysis at $-1.4 \mathrm{~V}$ vs. $\mathrm{Ag} / 0.1 \mathrm{M} \mathrm{Ag}{ }^{+}$. Initial solution composition: $4 \mathrm{mM} \mathrm{Co}(\text { bipy })_{3} 2+, 5.7 \mathrm{M}$ ally! chloride, 0.2M TEAP. Other conditions as in Fig. 5.

of the intense blue color of the solution. Gas chromatographic analysis showed that all of the allyl chloride had been consumed and about $50 \%$ had been converted to a 1,5-hexadiene. Cyclic voltammograms of the resulting solution were indistinguishable from those obtained with a fresh solution of $\mathrm{Co}$ (bipy) ${ }_{3}{ }^{2+}$.

The cobalt catalyzed reduction of allyl chloride may also be carried out chemically using borohydride as the reductant since sodium borohydride rapidly reduces $\mathrm{Co}$ (bipy) 3 or $2^{2+}$ to $\mathrm{Co}$ (bipy) 3 or $2^{+}$.

\section{Discussion}

The reduction of allyl chloride in the absence of cobalt complexes at mercury electrodes occurs near $-2.6 \mathrm{~V}$ ( vs. $\mathrm{Ag} / 0.1 \mathrm{M} \mathrm{Ag}{ }^{+}$) in nonaqueous solvents and the polarographic limiting current corresponds to two electrons per molecule of allyl chloride (3). Nevertheless, the primary reduction product of nonexhaustive electrolysis in which the concentration of allyl chloride is maintained relatively high is 1,5 -hexadiene (4) which requires only one electron per allyl chloride molecule (1-propene is also formed). These results have been rationalized (4) in terms of a chemical reaction between allyl chloride and allyl carbanion, the primary, two-electron reduction product.

In exhaustive, controlled potential electrolysis of $10 \mathrm{mM}$ solutions of allyl chloride with platinum electrodes we obtained rapid, clean, two-electron reduction at $-2.6 \mathrm{~V}$ in acetonitrile which we presume leads to the quantitative formation of 1-propene (no 1,5-hexadiene was detected by gas chromatography). The essential difference produced by the addition of cobalt-2,2'-bipyridine complexes to the electrolysis solution is the conversion from the two-electron reduction which yields allyl carbanion to a one-electron reduction yielding the allyl radical. The Scheme summarizes the proposed course of the reduction of allyl chloride both in the presence and in the absence of cobalt-2,2'-bipyridine complexes. 


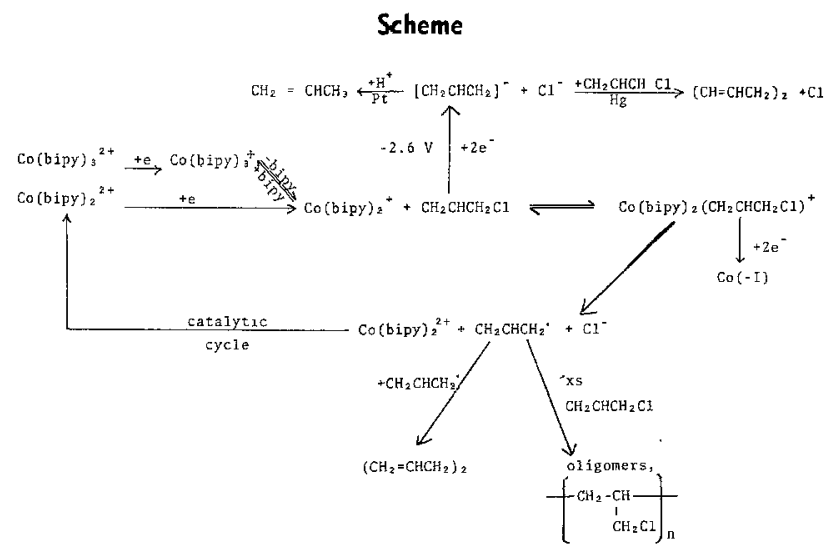

In the absence of cobalt a very negative electrode potential $(-2.6 \mathrm{~V})$ is required for the reduction of allyl chloride but quantitative reduction can be achieved at $-1.4 \mathrm{~V}$ in the presence of $\mathrm{Co}$ (bipy) $3_{3}{ }^{2+}$ (or Co (bipy) $2^{2+}$ ). In the catalytic cycle which leads to the reduction, $\mathrm{Co}$ (bipy) ${ }_{3}^{2+}$ is regenerated and this accounts for the enhancement of the reduction wave for this complex in the presence of allyl chloride (Fig. 1-3).

The proposition that the catalytic reduction involves an intermediate in which the allyl chloride is bonded to a complex of cobalt(I) and 2,2'-bipyridine is supported by several pieces of evidence: (i) Addition of 2,2 -bipyridine inhibits and eventually eliminates the catalytic reduction of allyl chloride (Fig. 2) even though the production of $\mathrm{Co}(\text { bipy })_{3}+$ is not impeded; (ii) the rate of the catalyzed reduction (as measured by the extent of the enhancement of the cobalt(II) reduction wave in cyclic voltammograms) is much higher when $\mathrm{Co}$ (bipy) ${ }_{2}{ }^{+}$is used as catalyst (Fig. 3); (iii) the new voltammetric wave at $-1500 \mathrm{mV}$ produced by the addition of allyl chloride to solutions of $\mathrm{Co}$ (bipy) $3^{2+}$ resembles in all regards the similar wave produced by acrylonitrile which is believed (1) to correspond to a stable, $\pi$-bonded, mixed complex in which the cobalt( $\mathrm{I})$ is reduced to cobalt $(-\mathrm{I})$. With acrylonitrile, the mixed complex is stable toward an intramolecular oxidation-reduction reaction, but with the more readily reducible allyl chloride the mixed complex decomposes as depicted in the Scheme.

The release of chloride anion in the decomposition of the mixed complex was confirmed by the appearance of an anodic wave at $+400 \mathrm{mV}$ when a mercury electrode was used to record cyclic voltammograms. That the other initial decomposition product is allyl radical is supported by the coulometric data (Table I). One electron per allyl chloride molecule is consumed in controlled potential electrolyses at ratios of allyl chloride to cobalt near unity. In addition, the appearance of oligomers of allyl chloride at higher ratios (Table I) is a strong indication that allyl radicals are present since the polymerization of allyl chloride requires radical (or cationic) initiation (5).

A rival mechanism for the decomposition of the mixed complex might involve the formation of a $\pi-$ allyl anion complex of $\mathrm{Co}$ (III) similar to a species prepared by Bercaw et al. (6) from reaction of allyl alcohol with a mixed cyano and phosphine complex of cobalt (I). Such a reaction sequence seems less attractive for the cobalt 2,2'-bipyridine complexes because Co (bipy) $2^{3+}$ is a rather strong oxidant $\left(E_{\mathrm{f}} \sim-70 \mathrm{mV}\right.$ vs. $\mathrm{Ag} / 0.1 \mathrm{M} \mathrm{Ag}{ }^{+}$) and allyl anion is so powerful a reductant $\left(E_{1 / 2} \sim-2.0 \mathrm{~V}\right.$ vs. $\left.\mathrm{Ag} / 0.1 \mathrm{M} \mathrm{Ag}{ }^{+}\right)$(3) that rapid decomposition of such a complex to Co (bipy) $2_{2}^{2+}$ and allyl radical would be favored unless extraordinary stability were attributed to the bond between cobalt (III) and the $\pi$-allyl anion.

\section{Conclusions}

The primary purpose of this communication was to point out the remarkable effectiveness of cobalt-2,2'bipyridine complexes as catalysts in the electroreduction of allyl chloride. The potential where the reduction commences becomes $c a .1 .2 \mathrm{~V}$ less negative in the presence of the catalyst. The mechanism proposed for the catalysis includes the initial formation of a $\pi$-complex between allyl chloride and $\mathrm{Co}$ (bipy) ${ }_{2}{ }^{+}$which parallels the recently described coordination chemistry of $\mathrm{Co}$ (bipy) ${ }_{2}{ }^{+}$and acrylonitrile (1). In the latter case, the lower reactivity of acrylonitrile terminates the reaction sequence with the coordination but with allyl chloride an intramolecular oxidation-reduction reaction ensues. Allyl bromide is itself reduced at potentials only slightly more negative than that at which Co (bipy) $3^{2+}$ is reduced but addition of the latter does result in the catalyzed reduction of allyl bromide.

The ability of a number of activated olefins to form $\pi$-bonds with low valent cobalt-2,2'-bipyridine complexes (1) suggests that these complexes may serve a general role as catalytic agents for the reduction of sufficiently activated (e.g., allyl chloride but not allyl alcohol or acrylonitrile) coordinated molecules.

\section{Acknowledgments}

This work was supported by the National Science Foundation. A Chaim Weizmann Fellowship supplied partial support for S.M.

Manuscript submitted Sept. 12, 1977; revised manuscript received ca. March 25, 1978.

Any discussion of this paper will appear in a Discussion Section to be published in the June 1979 JourNac. All discussions for the June 1979 Discussion Section should be submitted by Feb. 1, 1979.

\section{REFERENCES}

1. S. Margel, W. Smith, and F. C. Anson, This Journal, 125, 241 (1978)

2. N. Tanaka and $Y$. Sato, Bull. Chem. Soc. Jpn., 41, 2059 (1968)

3. J. P. Petrovich and M. M. Baizer, Electrochim. Acta, 12, 1249 (1967).

4. M. M. Baizer and J. L. Chruma, J. Org. Chem., 37, 1951 (1972)

5. E. Schildknecht, "High Polymers," Vol. XXVIII, p. 156, 707, Wiley Interscience, New York (1973).

6. J. E. Bercaw, L. Y. Goh, and J. Halpern, J. Am. Chem. Soc., 94, 6534 (1972). 